\title{
Non-Reflexive Logical Foundation for Quantum Mechanics
}

\author{
N. C. A. DA $\operatorname{Costa}^{1}$ and C. DE Ronde ${ }^{2}$ \\ 1. Universidade Federal de Santa Catarina - Brazil \\ 2. CONICET - Argentina \\ Center Leo Apostel (CLEA) and \\ Foundations of the Exact Sciences (FUND) \\ Brussels Free University - Belgium
}

\begin{abstract}
On the one hand, non-reflexive logics are logics in which the principle of identity does not hold in general. On the other hand, quantum mechanics has difficulties regarding the interpretation of 'particles' and their identity, also known in the literature as 'the problem of indistinguishable particles'. In this article, we will argue that non-reflexive logics can be a useful tool to account for such quantum indistinguishability. In particular, we will provide a particular non-reflexive logic that can help us to analyze and discuss this problem. From a more general physical perspective, we will also analyze the limits imposed by the orthodox quantum formalism to consider the existence of indistinguishable particles in the first place, and argue that non-reflexive logics can also help us to think beyond the limits of classical identity.
\end{abstract}

Keywords: identity, non-reflexive logic, interpretation of quantum mechanics. PACS numbers: 02.10 De

\section{Introduction}

It is well known in the literature that the notion of 'particle' in quantum mechanics (QM) faces serious difficulties when analyzed form a formal perspective. In particular, there are experts that defend the thesis that the notion of identity (or that of equality) is meaningless with reference to quantum particles or, at least, that it has to be restricted or modified in some sense 
(see for example: [18, p. 335], [2, p. 493-495] and [20, p. 248]). Our aim, in this paper, is to treat the problem of identity in QM from the perspective of non-reflexive logics. We introduce, in the first section, such kind of logics. In the second section, we formulate a particular non-reflexive logic. In the third section, it is outlined how the problem can be handled within the scope of the presented non-reflexive logic. In section four we analyze the constraints of the quantum formalism to discuss in terms of 'quantum particles' and argue that non-reflexive logics — which can allow us to bypass identity "right from the start" - are a formal tool that could help us to develop a different ontology from that of 'particles' - which, quite independently of the many efforts, does not seem to fit the orthodox formalism of QM.

\section{Non-Reflexive Logic}

Non-reflexive logic, in a wide sense, is a logic in which the relation of identity (or equality) is restricted, eliminated, replaced, at least in part, by a weaker relation, or employed together with a new non-reflexive implication or equivalence relation.

In classical logic, one of the basic principles is the so called principle of identity (PI), expressing the reflexive property of identity, whose usual formulation is as follows:

$$
x=x
$$

or

$$
\forall x(x=x)
$$

where $x$ is a first order variable. There are other versions in higher-order logic, in which appear higher order variables. There are also propositional formulations of the principle:

$$
p \rightarrow p \quad(p \text { implies } p)
$$

or

$$
p \leftrightarrow p \quad(p \text { is equivalent to } p)
$$

where $p$ is a propositional variable. If propositional quantification is allowed, then we have other forms of the principle: 


$$
\forall p(p \rightarrow p)
$$

as well as:

$$
\forall p(p \leftrightarrow p)
$$

Some of the above principles are not in general valid in non-reflexive logics. They are total or partially eliminated, restricted, or not applied to the relation that is employed instead of identity. Several are the motivations for the development of non-reflexive logics. For instance, the following:

1. Wittgenstein in his Tractatus, discussed the (possible) elimination of the relation of identity from logic (cf. $[27,7])$.

2. The propositional logic of causal implication is such that this kind of implication does not satisfy the propositional principle of causal identity [12].

3. From the logical and philosophical perspectives it is necessary to clarify the real meaning of the relation of identity in all levels of the logical hierarchy. In this way, as non-Euclidean geometries - independently of their intrinsic relevance - contribute to the better understanding of the very structure of Euclidean geometry, non-reflexive logic might helps us to explain the nature of identity and its principles. For instance, the obstacles to the construction of a convenient semantics for some of these logics (e. g., proper definitions of name, denotation, plurality, etc.) makes it easier to perceive their status as formalisms or as tools to cope with different problems. The systematic study of non-reflexive logic also makes evident how ordinary language is deeply involved with identity.

4. It seems appropriate to observe that Russell and Whitehead in their Principia Mathematica did already elaborate a theory of description that at least formally is non-reflexive [26]. Consequently, non-reflexive logical ideas were present in some context of classical logic, what may be a little surprising for most people. But the first reference to nonreflexive logic as a new form of (mathematical) logic, are to be found in $[4,5]$. 
5. Various authors, as referred to in the introduction to this paper, believe that identity is not in harmony with the foundations of quantum physics.

In the present paper we are concerned mainly with the last point. More specifically, with the possible solution provided by non-reflexive logics to the question of indistinguishable particles and to the possibility to, assuming a non-reflexive formalism "right from the start", develop an ontology which matches a non-reflexive formalism and escapes the presupposed ontology of particles.

\section{Non-Reflexive Set Theory $(Z F R)$}

In this section we construct a system of non-reflexive $\operatorname{logic} Z F R$, incorporating with a set theory, to the foundation of non-relativistic QM. Our auxiliary starting point is the usual Zermelo-Frenkel system of set theory with Urelemente (see, for example, [3]). Urelemente are the objects that are not sets. This system is denoted by $Z F U$. The language of $Z F U$ is built as follows:

1. The primitive symbols are $\epsilon$ (elementhood), $\mathcal{C}$ (a monadic predicate constant; $\mathcal{C}(x)$, where $x$ is a variable, means that $x$ is a set), and the common primitive logical symbols of a systematization of the classical first-order predicate calculus with identity (equality). On these symbols, see [21] or [25], whose terminology, notations, etc., we follow with some obvious adaptations.

2. The syntactic concepts such as those of terms, formula, sentence, free variable, etc., are also adaptations of the two just cited books.

3. Deductive structure of $Z F U$ : the notions of axiom, axiom scheme, postulate (axiom or axiom scheme, as well as primitive rule of inference), etc., are those of the cited books with clear changes.

We write $\left(\forall_{\mathcal{C}} x\right)(\ldots)$ instead of $(\forall x)(\mathcal{C}(x) \rightarrow \ldots)$ and $\left(\exists_{\mathcal{C}} x\right)(\ldots)$ instead of $(\exists x)(\mathcal{C}(x) \& \ldots)$.

The specific postulates of $Z F U$ are the following:

(A.1) $\left(\forall_{\mathcal{C}} x\right)\left(\forall_{\mathcal{C}} y\right)((\forall z)(z \in x \leftrightarrow z \in y) \rightarrow x=y)$. 
(A.2) $(\forall x)(\forall y)\left(\exists_{\mathcal{C}} t\right)(\forall z)(z \in t \leftrightarrow z \in x \vee z \in y)$.

(A.3) $\left(\forall_{\mathcal{C}} x\right)\left(\exists_{\mathcal{C}} y\right)\left(\forall_{\mathcal{C}} t\right)(t \in y \leftrightarrow t \subseteq x)$.

If $F(x)$ is a formula, $x, y$ and $z$ are distinct variables and $y$ does not occur free in $F(x)$, we have:

(A.4) $\left(\forall_{\mathcal{C}} z\right)\left(\exists_{\mathcal{C}} y\right)(\forall x)(x \in y \leftrightarrow F(x) \& x \in z)$

$(\mathrm{A} .5)\left(\exists_{\mathcal{C}} t\right)(\forall x)(x \notin t)$.

(A.6) $\left(\forall_{\mathcal{C}} x\right)\left((\forall y)(y \in x \rightarrow \mathcal{C}(y)) \rightarrow\left(\exists_{\mathcal{C}} z\right)(\forall t)(t \in z \leftrightarrow(\exists v)(v \in x \& t \in\right.$ $v))$.

If $F(x, y)$ is a formula, and the variables satisfy evident conditions, we have:

$$
(\forall x)(\exists ! y) F(x, y) \rightarrow\left(\forall_{\mathcal{C}} u\right)\left(\exists_{\mathcal{C}} v\right)(\forall y)(y \in v \leftrightarrow(\exists x)(x \in u \& F(x, y))) .
$$

(A.8) $\left(\exists_{\mathcal{C}} z\right)(\emptyset \in z \&(\forall x)(x \in z \rightarrow x \cup\{x\} \in z))$.

(A.9) $\left(\forall_{\mathcal{C}} x\right)\{(\forall y)(y \in x \rightarrow \mathcal{C}(y)) \&(\forall y)(\forall z)(y \in x \& z \in x \rightarrow(y \cap z=$ $\left.\emptyset \& y \neq \emptyset)) \rightarrow\left(\exists_{\mathcal{C}} u\right)(\forall y)(\exists v)(y \in x \rightarrow(y \cap u=\{v\}))\right\}$.

Remark. It is possible to adjoin the following postulate of regularity to $Z F U$ (although we shall not do so here):

$$
\left(\forall_{\mathcal{C}} x\right)(x \neq \emptyset \&(\forall y)(y \in x \rightarrow \mathcal{C}(y)) \rightarrow(\exists z)(z \in x \& z \cap x=\emptyset)
$$

The above schemes and axiom schemes are those of $Z F$ with Urelemente (see, for instance, [16]). For example, (A.4) is the axiom scheme of separation and (A.10) is the axiom of regularity. We need, in addition, one more axiom:

(A.11) $\exists_{\mathcal{C}} y \forall x(x \in y \leftrightarrow \neg \mathcal{C}(x))$.

which says that the totality of Urelemente constitutes a set.

The symbol $\{x: F(x)\}$, where $x$ is a variable and $F(x)$ is a formula, denotes the set of all objects that satisfy $F(x)$, if this set does exist, that is, if $\exists_{\mathcal{C}} y \forall x(x \in y \leftrightarrow F(x))$ is a theorem of $Z F U$. We put, by definition, that $U=\{x: \neg \mathcal{C}(x)\}$. It is not difficult to see that $Z F U$ is a strong set theory, in which it is possible to develop, with clear adaptations, the entire contents of usual Zermelo-Frenkel system of set theory (see, for example [3] and $[16])$. 
We enrich $Z F U$ with the introduction of the finite and non-empty sets $m, m_{1}, m_{2}$ and $M$, under the following conditions: 1) $U=m \cup M$; 2) $m=$ $m_{1} \cup m_{2}$;3) $m_{1} \cap m_{2}=\emptyset$; 4$) m \cap M=\emptyset$. Informally, $m$ is the set of quantum objects $m_{1}$ and $m_{2}$, are two kinds of quantum objects, and $M$ is the set of classical objects. (In the general case, we have $m=m_{1} \cup m_{2} \cup m_{3} \cup \ldots \cup$ $m_{k}, 0<k<\omega ; m_{1}, m_{2}, \ldots, m_{k}$ are mutually exclusive sets).

The next developments of $Z F U$ are informally presented. Small Greek letters stand for ordinals and the class of all ordinals is represented by Ord. $Z F$ is Zermelo Frenkel theory.

Definition 2.1 By transfinite induction we put ( $\mathbb{P}$ is the power-set of $x)$ : $V_{0}=U=m_{1} \cup m_{2} \cup M, V_{1}=\mathbb{P}\left(V_{0}\right), V_{2}=\mathbb{P}\left(V_{1}\right), \ldots, V_{n+1}=\mathbb{P}\left(V_{n}\right), \ldots$, $V_{\omega}=\bigcup_{\beta<\omega}\left(V_{\beta}\right), \ldots, V_{\omega+n+1}=\mathbb{P}\left(V_{\omega}+n\right), \ldots, \mathbb{V}=\bigcup_{\alpha \in O r d}\left(V_{\alpha}\right)$.

Definition 2.2 Analogously, the hierarchies $V_{\alpha}^{\prime}$ and $V_{\alpha}^{\prime \prime}$, with $V_{o}^{\prime}=M$ and $V_{o}^{\prime \prime}=\phi$, are defined. We put: $\mathbb{V}^{\prime}=\bigcup_{\alpha \in O r d}\left(V_{\alpha}^{\prime}\right)$ and $\mathbb{V}^{\prime \prime}=\bigcup_{\alpha \in O r d}\left(V_{\alpha}^{\prime \prime}\right)$.

Definition 2.3 If $x \in V_{0}$, then $\operatorname{rank}(x)$ is the least $\alpha$ such that $x \in V_{\alpha}$. Similarly, we define $\operatorname{rank}^{\prime}(x)$ and $\operatorname{rank}^{\prime \prime}(x)$.

Theorem 2.4 $\mathbb{V}^{\prime \prime} \subset \mathbb{V}^{\prime} \subset \mathbb{V}$.

Proof: Immediate.

Theorem 2.5 $x \in \mathbb{V}^{\prime \prime} \rightarrow \mathcal{C}(x) ; x \in \mathbb{V}^{\prime \prime} \rightarrow x \subset \mathbb{V}^{\prime \prime}$.

Proof: By induction on the $\operatorname{rank}^{\prime \prime}(x)$ as it is usual.

Theorem 2.6 If $x \in \mathbb{V}^{\prime \prime}$, then $\operatorname{rank}(x)=\operatorname{rank}^{\prime}(x)=\operatorname{rank}^{\prime \prime}(x)$.

Proof: By induction on the $\operatorname{rank}^{\prime \prime}(x)$.

Theorem 2.7 $\mathbb{V}$ is an inner model of $Z F U$.

Proof: By transfinite induction, as in the case of $Z F$.

Theorem $2.8 \mathbb{V}^{\prime \prime}$ is an inner model of $Z F$ in $Z F U$.

Proof: Analogous to the proof of the preceding theorem.

Theorem 2.9 If $Z F M$ is the theory $Z F$ with the set $M$ of Urelemente, then $\mathbb{V}^{\prime}$ is an inner model of $Z F M$ in $Z F U$.

Proof: As the proof of the preceding theorem. 
Definition 2.10 The binary relation $\equiv$ is defined on $\mathbb{V}$ by transfinite induction through the following clauses:

Initial clauses, defining $\equiv$ on $\left.V_{0}: 1\right)$ if $x, y \in m_{i}, 1 \leq i \leq 2$, then $x \equiv y$ and $y \equiv x$; 2) if $x \in m_{1}$ and $y \in m_{2}$, then $x \not \equiv y$ and $y \not \equiv x$ ( $x \not \equiv y$ is the negation of $x \equiv y$; 3) if $x \in m_{2}$ and $y \in m_{1}$, then $x \not \equiv y$ and $y \not \equiv x$; 4) if $x \in m$ and $y \in M$, then $x \not \equiv y$ and $y \not \equiv x$; 5) if $x, y \in M$, then $x \equiv y$ if and only if $x=y$; 6) if $x \in V_{0}$ and $y$ is a set, then $x \not \equiv y$ and $y \not \equiv x$.

Inductive clauses: 1) if $x$ and $y$ are sets, $x \in \mathbb{V}^{\prime}$ and $y \notin \mathbb{V}^{\prime}$, then $x \neq \equiv y$ and $y \not \equiv x$; 2) if $x$ and $y$ are sets and $x, y \in \mathbb{V}^{\prime}$, then $x \equiv y$ if and only if $x=y$; 3) if $x$ and $y$ are sets and $x, y \notin \mathbb{V}^{\prime}$, then $x \equiv y$ and $y \equiv x$ if and only if:

$\forall z(z \in x \rightarrow \exists w(w \equiv z \wedge w \in y)) \wedge \forall t(t \in y \rightarrow \exists v(v \equiv t \wedge t \in x)) \wedge \operatorname{card}(x)$ $=\operatorname{card}(y)$, where $\operatorname{card}(x)$ is the cardinal number of $x$.

Theorem 2.11 The relation $\equiv$ is well defined on $\mathbb{V}$.

Proof: In effect, $x \equiv y$ implies that $\operatorname{rank}(x)=\operatorname{rank}(y)$, ordinal number that we denote by $\alpha$. Then, if $\equiv$ is defined for all elements $t$ of $\mathbb{V}$ of $\operatorname{rank}(t)<\alpha$, it easily follows that $x \equiv y$ is also defined.

Definition 2.12 If $x$ and $y$ are sets, then we put:

$x \prec y \stackrel{\text { def }}{=} \forall z(z \in x \rightarrow \exists \omega(\omega \equiv z \wedge \omega \in y))$

$x \cong y \stackrel{\text { def }}{=} x \prec y \wedge y \prec x$

$x \prec y$ is read ' $x$ is a quantum subset of $y$ '; $x \cong y$ is read ' $x$ and $y$ are weakly quantum equivalent'.

$Z F R$ is $Z F U$ enriched by the individual constant $m_{1}, m_{2}, m$ and $M$, and the predicate constant $\equiv$. Informally, $m$ is the set of quantum objects, $M$ is the set of classical objects, and $m_{1}$ and $m_{2}$ are two sorts of quantum objects. The relation $\equiv$, between elements $m$, means quantum indistinguishability or (quantum) equivalence; $\equiv$ is extended to all pairs of elements of $\mathbb{V}$ according to its intended meaning. By definition of $Z F R$, its specific postulates are those of $Z F U$ conveniently adapted.

We now present a list of theorems of $Z F R$ whose proofs are not difficult. Every permutation p of $m_{1}$ (or of $m_{2}$ ) induces a transformation $\bar{p}$ of any set of $\mathbb{V}$ into $\mathbb{V}$. Such permutations are called quantic permutations.

Theorem 2.13 If $p$ is a quantic permutation and $x$ and $y$ are sets of $\mathbb{V}$ such that $x \equiv y$, then $\bar{p}(x) \equiv \bar{p}(y)$. 
Proof: $\quad$ By induction on the rank of $\mathbb{V}$, taking into account the definition of $\equiv$.

Corollary: Under the condition that $x, y \in \mathbb{V}^{\prime}$, we have: $x=y \leftrightarrow \bar{\beta}(x)=$ $\bar{\beta}(y)$.

Where $\bar{\beta}$ are formulas.

Theorem 2.14 $x \equiv x ; x \equiv y \rightarrow y \equiv x ;(x \equiv y \wedge y \equiv z) \rightarrow x \equiv z ; x=y \rightarrow$ $x \equiv y$.

Theorem 2.15 If $x$ and $y$ are sets, then:
1. $x \equiv y \leftrightarrow \forall z(z \in x \rightarrow \exists w(w \equiv z \wedge w \in y))$
2. $x, y \in \mathbb{V}^{\prime} \rightarrow(x \equiv y \leftrightarrow x=y)$
3. $x \in y \rightarrow \operatorname{rank}(x)<\operatorname{rank}(y)$
4. $x \equiv y \rightarrow \operatorname{card}(x)=\operatorname{card}(y)$
5. $x \equiv y \rightarrow x \cong y$
6. $x \equiv y \leftrightarrow(x \cong y \wedge \operatorname{card}(x)=\operatorname{card}(y))$

Definition $2.16 x \bar{\in} y \stackrel{\text { def }}{=} \exists z(z \equiv x \wedge z \in y)$.

Definition 2.17 Let $F$ be a formula of $Z F R$. We denote by $F^{*}$ the formula obtained from $F$ by replacing all occurrences of $=b y \equiv$, and all occurrences of $\in$ by $\bar{\epsilon}$.

Theorem 2.18 If $F$ is a theorem of $Z F R$, then $F^{*}$ is also a theorem of the same system.

Proof: By induction on the length of $F$.

Theorem 2.19 Let us suppose that it is added to ZFR new individual constants to name, biunivocally, the elements of $m_{1}$ and new individual constants to name biunivocally the elements of $m_{2}$, and that $p$ is a permutation of the names of the elements of $m_{1}$ (or of $m_{2}$ ). The new system so obtained is denoted by $Z F R^{-}$. Under these hypothesis, one has: if $F$ is a theorem of $Z F R^{-}$and $F^{-}$is obtained from $F$ by replacing the name $K$ of any element of $m_{1}$, appearing in $F$, by $p(k)$, then $F^{-}$is also a theorem of $Z F R^{-}$.

Proof: Trivial. 
$Z F R$ is a non-reflexive system since it contains a relation $\equiv$, of quantum equivalence (or quantum indistinguishability), which is used in various contexts of QM to replace identity. In some cases, as those referred to in the introduction to the paper, $\equiv$ is really important, and identity, for some authors lose, at least in part, its meaning. However, here, we shall employ $Z F R$ basically as a classical set theory, although one could try to handle $Z F R$, as a strict non-reflexive logic, to eliminate identity in connection with quantum particles (see, for example, [17] and also [1, 10, 15, 23]). Therefore, one may say that $Z F R$ is a kind of 'classical non-reflexive' logic.

\section{Indistinguishability and Non-Reflexive Logic}

In this section, we outline how non-relativistic QM may be founded on $Z F R$. In $[18$, p. 335] Ghirardi states that:

"Once we have understood that it is impossible to individualize the indistinguishables, we are let to assuming the impossibility of such operation itself as a criterion of identity: two elementary particles will be proclaimed identical when we must recognize that no physical procedure exists that permits us to distinguish them from each other, or in other words, when all the physical implications about the system in question are unchanged when we imagine 'switching' the constituents"

This is clearly interpretable as saying that 'identity' in QM really means that two elementary particles of the same sort are identical in the sense they are physically indiscernible, i.e. from our perspective, they are quantum equivalent. So, we must distinguish, carefully, between logical identity $(=)$ and quantum equivalence ( $\equiv)$. Some physicists call ' $\equiv$ ' physical identity. Notice that the notion of 'switching' only makes sense in an ontology which presupposes individuals that can be interchanged and have some kind of identity. Thus if we accept the possibility of 'switching' the question of individuals with no identity cannot be posed. One can also insist, following Holland [20], on the role of logical identity in QM, although $\equiv$ is a derived, but relevant concept.

We have, in effect, two major concerns in connection with logical identity and quantum equivalence:

(1) Substitutivity: If $a$ and $b$ are quantum equivalent, then, in any physical system, $a$ can be replaced by $b$ without physically changing the system. 
(2) The existence of quantum objects may be quantum equivalent but not logically identical - since identity is considered by many physicists as meaningless.

(1) and (2) are such that we may have (1) although not (2), and (1) and (2).In this second case, since $Z F R$ does contain identity, this relation may be seen as an "improper" relation, purely symbolic, when referring to quantum objects, more or less as points at infinity in Euclidian geometry. Both situations are accommodated in $Z F R$. This is one of the main reasons we call $Z F R$ a 'classical non-reflexive logic'.

After the presentation of quantum structures and their postulates, theorems 2.13 and 2.19 remain valid; therefore, quantum equivalence is a congruence relation satisfying the laws of substitutivity. Here, we shall treat a particular form of QM, in which both = and $\equiv$ are used and are basic, which mirrors what most physicists do.

The basic orthodox interpretation of QM is that the state of a quantum system, say a particle of mass $m$, is given by a complex valued function $\Psi$, the so called 'wave function' which depends on the three space coordinates $\Re^{3}$ and time. Newtonian space-time constitutes the frame involving quantum systems. In addition, there exists another function $\Phi$, the Fourier transform of $\Psi$, conveniently normalized, that furnishes the momentum. For $n$ particles, $\Psi$ and $\Phi$ are complex valued functions defined on $\Re^{3 n}$ and time. The Schrödinger equation characterizes the dynamics. $\Psi$ might be viewed as a vector in a Hilbert Space (a space of functions), and the probability for the distribution of particles is given by the known Born rule. The states of a quantum system are vectors of the Hilbert space and the observables self-adjoint operators in the same space, etc.

Taking into account such interpretation, we introduce the quantum structures, which are mathematical structures, as follows:

$$
e=<C, \Re^{3 n} \times \Re, H, \Psi, P>.
$$

Where the following postulates are satisfied:

1. $C$ is a set of $n(n \in \omega)$ elements of the set $m$, set of the quantum objects;

2. $\Re^{3 n}$ is the Cartesian product of $\Re^{3}$ by $\Re^{3} n$ times and $\Re$ is the set of reals that represent instants of time; $\Re^{3 n}$ is the configuration space. 
3. $H$ is a complex Hilbert space, that is separable, etc.;

4. $\Psi$ is a vector of $H$, called the 'wave function', given the state of the system, and defined on $\Re^{3 n} \times \Re(\times$ is the cartesian product);

5. $P$ is a function defined by the Born rule;

6. The dynamics of the system is summarized by the Schrödinger equation.

Other relevant notions such like momentum, $\Phi$, angular momentum, free particle, scattering, etc., are all definable. However, there are other, extra concepts, such as spin, and extra postulates, for instance Pauli's principle, that need to be adjoined to cope with some questions. Details may be seen in works on the subject, among which we mention [19] and [24].

Mathematically, QM is the theory of quantum structures of species $e$. This mathematical theory of certain kind of structures (cf. $[7,11])$ is what the physicist tries to relate to physical experience.

It is quite clear that theorems 2.13 and 2.19 remain valid in the mathematical treatment of QM based on $Z F R$. Thus there are two main routes to deal with identity in QM under the supposition that $Z F R$ is its underlying logic:

1. We can employ both identity and quantum equivalence in QM with reference to quantum objects;

2. We can give emphasis on quantum equivalence in connection with quantum objects in which case the recourse to identity, especially its reference to 'classical' objects, would be a theoretical device to simplify some aspects of QM. Clearly, this presupposes the consistency of QM based on ZFR.

However, there are other reasons to found QM on a non-reflexive logic. In the next section we make some comments on the connections between ontology and identity in QM that may constitute the starting point of a new no-reflexive foundation for this science. We intend to develop the more technical parts of this paper in future works. The philosophical aspects will be also treated in detail. 


\section{Beyond 'Quantum Particles'?}

As we mentioned above - leaving aside instrumentalist interpretations which deny the need of an interpretation - there are two main strategies to account for the problem of indistinguishable particles in QM. We can either start our analysis assuming that QM talks about some strange kind of 'particles' which do not seem to follow partly or completely the PI - and then try to make sense of the formalism, or we can accept that we do not know what QM is talking about, start our investigation from the formalism itself and try to find an appropriate ontology which is not restricted to the notion of 'particle'. In the following section we provide arguments against the first line of analysis and call the attention to the importance of non-reflexive logics within the second proposed line of research.

The two authors of this paper believe that within physical theories there is a tight interrelation between logic and ontology which cannot be bypassed by either side. There are many logics and possible ontologies. A given ontology is always related to a specific kind of logic and, vice versa, a given logic might determine, at most, a restricted set of possible ontologies that can be coherently developed from the logical scheme. Thus, when attempting to interpret a theory, either starting from the formal logical perspective or from the ontological one, the physicist — who attempts to provide a physical representation of the theory - needs to call special attention to the fact that both ontology and logic must relate in a coherent manner. This interrelation must also be capable of allowing us to discuss about physical experience - e. g., the experience provided by Maxwell's theory regarding electromagnetic waves, the experience provided by Newtonian mechanics regarding physical particles and bodies, etc. One must be careful however, for it is in principle not necessarily true that from any logic one can develop a suitable ontology and, vice versa, that from every ontology one can coherently find a suitable logical scheme.

Within Aristotelian metaphysics the PI, together with the principle of existence and the principle of non-contradiction, constitute and determine not only classical logic but also the notion of 'entity' - of which the notion of 'particle' is a particular case. These principles play not only a logical but also an ontological role in Aristotle's architectonic. And it is not self evident that one can "leave aside" one of such constitutive principles and claim that one is still talking about the same ontology; it is not at all obvious that without the PI one can still talk about 'entities'. One must be cautious for if we attempt to provide a physical interpretation of a given formalism one also needs to 
be clear about what the formalism is talking about - e. g. 'particles', 'waves', 'fields', etc. Neglecting this interpretational fact can rapidly direct us, willingly or not, through the unwanted path of instrumentalism.

Pointing to 'something' at time $t_{1}$ relates, through the PI, to the same 'something' at time $t_{2}$. Thus it is the PI which allow us to state that we have the same 'something' through time. But even in one instant of time we run into trouble in case we naively assume there are a certain number of 'indistinguishable objects'. In such case their 'existence in space' — quite independently of time - makes them distinguishable, for any object with a definite position becomes distinct to another one in a different position. This means that if we are to take indistinguishability in ontological terms, we must accept that 'indistinguishable objects' cannot exist within spacetime. They must exist with a different "ontological support". But what is the meaning of 'a particle which does not exist in space nor time'? Can this be regarded as a 'physical particle'? Or is it space-time a necessary precondition for talking about particles? Does this notion make sense at all or is it just a logical game with no physical counterpart? The question remains if an 'entity with no identity' is a thinkable physical object or rather, just an oxymoron. But even leaving aside the problems to reconcile the PI with the quantum formalism there are other aspects of the formalism which go deeper against the presupposition of a 'particle ontology' for QM.

One can discuss individuality and identity in the case of the statistical properties of indistinguishable quanta, but we can also go a step further and claim that the failure of the applicability of the notion of individuality occurs in a more general frame. Indeed, it occurs within the whole structure of QM. Let us consider the set $\mathcal{L}$ of physical properties of a quantum system. The formalism of the theory associates to each physical magnitude a mathematical object - an operator, called "observable", over the Hilbert space of states of the system - and the Heisenberg principle states that not all magnitudes may possess definite values at the same time. This must not be interpreted as a consequence of our ignorance or of our inexact procedures to determine them. Only subsets of compatible magnitudes may simultaneously possess values. ${ }^{1}$ This is strongly different from the classical

\footnotetext{
${ }^{1}$ The indetermination of the values of incompatible pairs is a matter of principle. In fact, it is one of the fundamental physical principles from which the formal structure of the theory may be derived. In mathematical terms, observables linked by the Heisenberg principle do not commute and thus, physical magnitudes obey a non-commutative algebra - technically, the projectors in which they decompose are structured in a modular lattice in the finite case.
} 
realm - where they are structured in a Boolean lattice - and thus there exist (Boolean) valuations of all propositions about physical magnitudes. The different algebraic structure of the quantum properties has as its counterpart the different meaning of the logical connectives among propositions regarding properties. Thus, if we naively try to interpret them as classical properties, as properties possessed by the system, we are faced to all kind of no-go theorems that preclude this possibility. Most remarkably is the Kochen-Specker (KS) theorem which explicitly shows the fact that within the formal structure of QM, it is not possible to jointly assign truth values to different not-disjoint subsets of mutually compatible properties [22]. This is a very strong impediment to get an image of quantum systems in some sense close to classical objects. One of us has claimed that the conclusion which must be driven from the KS theorem is that the quantum wave function cannot be conceived in terms of 'the state of an individual which possesses properties' [13]. The possible mathematical representations which expose the quantum wave function from different basis cannot be interpreted as related to properties which preexist (to measurement). Thus, the KS theorem shows the impossibility to unify the different representations in a unique and singular 'whole', in something which can be considered as a classical individual. As it has been analyzed in [14] one cannot naively assume that the choice of the context allows us to claim that classicality has been restored.

Following this line of thought, it could be argued that one should leave the door open to the possibility to consider an interpretation of QM which is not necessarily based on the ontology of 'particles'. If such possibility is granted and taken seriously, just in the same way paraconsistent logics might be an interesting tool for discussing a new interpretation of quantum superpositions [8], a radical Non-Reflexive Logic with no identity might be a perfect tool to advance in an interpretation that is not restricted by (classical) metaphysical presuppositions.

\section{References}

[1] Arenhart, J.R.B. and Krasue, D., 2012, "A discussion on quantum nonindividuality", journal of Applied Non-Classical Logic, 22, 105-124.

[2] Bohm, D., 1951, Quantum Theory, Dover, New York.

[3] Brignole, D. and da Costa, N., 1971, "On supernominal EhresmannDedcker universes", Math. Z, 122, 342-350. 
[4] da Costa, N. C. A., 1979, Ensaio sobre os Fundamentos da Lógica, HUCITEC.

[5] da Costa, N. C. A., 1986, "Aspectos de la lógica atual", in En Chile También hay Ciencia, J. Ioda, J. Melmick and S. Melmick (Eds.), pp. 221-240, Universidad de Chile, Santiago de Chile.

[6] da Costa, N. C. A., 1997, Logique Classique et Non-Classique, Masson, Paris.

[7] da Costa, N. C. A. and Bueno, O., 2009, "Non reflexive logics", Revista Brasilera de Filosofía, 232, 181-196.

[8] da Costa N. C. A. and de Ronde, C., 2013, "The Paraconsistent Logic of Quantum Superpositions", Foundations of Physics, 43, 845-858.

[9] da Costa, N.C.A. and Krause, D., 1999, "Set theoretical models for quantum systems", in Language, quantum, music, M. L. Dalla Chiara, R. Giuntin and F. Laudisa (Eds), 171-181, Kluwer.

[10] da Costa, N.C.A. , Krause, D., Arenhart, J.R.B. and Schinaider, J., 2012, "Sobre uma fundamentacao nao-reflexiva da mecanica cuantica", Scientiae Studia, 10, 71-104.

[11] da Costa, N.C.A. and Rodrigues, A. A. M., 2007, "Definability and Invariance", Studia logica, 86, 1-30.

[12] da Costa, N. C. A. and Routley, R., 1987, "Cause as an implication", Studia Logica, 47, 413-428.

[13] de Ronde, C., 2011, The Contextual and Modal Character of Quantum Mechanics: A Formal and Philosophical Analysis in the Foundations of Physics, $\mathrm{PhD}$ dissertation, Utrecht University.

[14] de Ronde, C., Freytes, H. and Domenech, G., 2014, "Interpreting the Modal Kochen-Specker Theorem: Possibility and Many Worlds in Quantum Mechanics", Studies in History and Philosophy of Modern Science, 45, 11-18.

[15] Domenech, G. Holik, F. and Krause, D., 2008, "Q-Spaces and the Foundations of Quantum Mechanics", Foundations of Physics, 38, 969-994.

[16] Fraenkel A. A. and Bar-Hillel, Y., 1958, Foundations of Set Theory, North-Holland, Amsterdam. 
[17] French, S. and Krause, D., 2006, Identity in Physics, Clarendon Press, Oxford.

[18] Ghirardi, g., 2005, Sneaking a Look to God's Cards, Princeton University Press, Princeton.

[19] Griffiths, D., 1996, Introduction to Quantum Mechanics, Mc Graw-Hill, New York.

[20] Holland, P. R., 1995, The Quantum Theory of Motion, Cambridge University Press, Cambridge.

[21] Kleene, S., 1952, Introduction to Metamathematics, North Holland, Amsterdam.

[22] Kochen, S. and Specker, E., 1967, "On the problem of Hidden Variables in Quantum Mechanics", Journal of Mathematics and Mechanics, 17, 59-87. Reprinted in Hooker, 1975, 293-328.

[23] Krause, D. and Arenhart, J.R.B., "Classical or Non-Reflexive Logics? A Case of Semantic Underdetermination", forthcoming.

[24] Liboff, R. L., 1997, Introductory Quantum Mechanics, Reading Mass, Addison Wesley, Massachusetts.

[25] Schoenfield, J., 1967, Mathematical Logic, Reading, Massachusetts.

[26] Whitehead, A. N. and Russell, B., 1910, Principia Mathematica, vol. 1, Cambridge University Press, Cambridge.

[27] Wittgenstein, L., 1988, Tractatus Logico-Philosophicus, (transl. by C. K. Odgen), Routledge and Kegan Paul, London. 